Note d'information

\title{
PRÉSENTATION DE L'ORGANISATION DE LA PECHE DES POISSONS MIGRATEURS ET DE LA PÊCHE DANS LES ESTUAIRES EN FRANCE PAR LES PROFESSIONNELS MARITIMES : LE SYSTÈME DES LICENCES « CIPE ».
}

\section{GUERNALEC}

Commission des poissons migrateurs et des estuaires (C.I.P.E.) du Comité National des Pêches Maritimes et des Elevages Marins ${ }^{1}$ (C.N.P.M.E.M.), 51 rue Salvador Allende, 92027 Nanterre Cedex, France.

Reçu le 22 septembre 1999

Accepté le 12 avril 2000
Received 22 September, 1999 Accepted 12 April, 2000

\section{RÉSUMÉ}

Depuis la fin des années 1970, l'exercice à titre professionnel de la pêche maritime des poissons migrateurs et de la pêche dans les estuaires en France fait l'objet d'une organisation spécifique à travers un système de licences limitatives de l'accès à la ressource mis en place par la profession elle-même, en liaison étroite avec les pouvoirs publics au plan national ; connu sous l'appellation de système des « licences CIPE ", ce dispositif contraignant d'encadrement des activités de pêche vise, dès l'origine, à réserver laccès des écosystèmes halieutiques estuariens aux seules petites unités de pêche côtières traditionnelles particulièrement dépendantes de la pêche des poissons amphihalins et dont l'économie constitue un maillon essentiel de l'aménagement du territoire et de la préservation des équilibres socio-économiques du littoral.

Après avoir souligné l'importance socio-économique des pêcheries maritimes des estuaires, en particulier de l'anguille-civelle «Anguilla anguilla en France, nous essayerons de montrer de quelle manière les marins-pêcheurs des estuaires ont su, dans le cadre d'ensemble de l'interprofession maritime et de ses compétences réglementaires dans le domaine de la protection des ressources halieutiques, instaurer ce dispositif de gestion propre aux pêcheries de poissons amphihalins; nous décrirons ensuite plus en détails les principes et modalités de fonctionnement du système des licences CIPE (champ d'application, contingentement, gestion par bassins etc...) et tenterons d'en montrer tout l'intérêt au regard des impératifs d'une exploitation rationnelle et responsable ainsi que des enjeux actuels de la lutte contre le braconnage et d'une meilleure protection des milieux aquatiques.

Mots-clés : poissons migrateurs, anguilles, estuaires, réglementation, licences, marins-pêcheurs.

\footnotetext{
'La Commission des poissons migrateurs et des estuaires (CIPE) fait partie des 12 Commissions spécialisées du Comité National des Pêches Maritimes et des Elevages Marins (C.N.P.M.E.M.), organisme créé par la loi $n^{2} 91.411$ du 2 mai 1991 « relative à l'organisation interprofessionnelle des pêches maritimes et des élevages marins (...) ". J.O.R.F. du 7 mai 1991, p. 6072. Depuis 1992 , cette Commission intervient aux lieu et place de lancien Comite interprofessionnel des poissons migrateurs et des estuaires (crée en 1978) aujourd'hui disparu.
} 


\title{
MIGRATING FISH ORGANISATION \\ IN FRANCE BY MARITIME AND ESTUARY PROFESSIONNAL FISHING THROUGH THE ACCESS LICENSING « CIPE ».
}

\begin{abstract}
Since the end of the 1970's, maritime migrating fish and estuary professional fishing in France have been organised through a system of restrictive access licensing devised by the profession itself. This licensing system was established in close cooperation with national authorities and is known as the system of "licences CIPE ». This restrictive scheme allows for supervision of fishing and aims to reserve the access of fishing ecosystems to small traditional coastal fishing units which are particularly dependant on migrating fishes and are an essential link in planning and preserving the socio-economic balance of coastlines.
\end{abstract}

Once we have emphasised the socio-economical importance of migratory species in France, -particularly with eel and glass-eel-, this paper will try to show how estuarine maritime fishermen have been able to use the framework of all French maritime fishing professions in their ability in matters of the protection and management of fishing resources in order to establish a specific licensing system. We also will describe the principles and modes of management of this licensing system, as currently implemented in France (application fields, restrictive licensing and decentralised management) and aim to demonstrate its pertinence to the current requirements for better protection of the environment and better fighting again poaching.

Key-words : migrating fish, eel, estuaries, regulations, licensing system, maritime fishermen.

\section{PROPOS LIMINAIRES : L'IMPORTANCE SOCIO-ÉCONOMIQUE DE LA PÊCHE DANS LES ESTUAIRES}

La pêche professionnelle maritime des poissons migrateurs et des estuaires représente en France plus de 1100 entreprises de pêche réparties sur le littoral de la Manche et de l'océan Atlantique auxquelles il faut associer près de 500 marins-pêcheurs pratiquant dans les lagunes et étangs du littoral méditerranéen.

L'espèce « anguille-civelle " constitue la composante essentielle de l'exploitation halieutique des zones estuariennes, aussi bien le long de la côte Atlantique pour la capture de la civelle qu'en mer Méditerranée pour l'exploitation de l'anguillette et de l'anguille? Dans ces deux zones, on estime qu'en moyenne chaque entreprise de pêche peut assuret entre $50 \%$ à $75 \%$ de ses revenus grâce à cette seule ressource ${ }^{3}$. L'exploitation de l'anguille-civelle est donc absolument vitale pour la survie de ces petites entreprises de pêche.

L'anguille adulte étant encore assez peu valorisée en France, l'importance de la filière "anguille " est due, essentiellement, à l'exploitation de la civelle sur la côte Atlantique (de l'ordre de 300/350 tonnes par an); celle-ci a connu, surtout depuis l'amnée 1995, une augmentation significative de son prix de vente en raison de l'ouverture de

\footnotetext{
${ }^{2}$ La pêche de la civelle est interdite sur les côtes françaises de la mer Méditerranée.

${ }^{3}$ Pour une vue d'ensemble de l'importance socio-économique de la pêche professionnelle des poissols amphihalins, voir les actes des Assises nationales de la pêche professionnelle organisées par le C.N.P.M.E.M. C.I.P.E. et la Coordination nationale de la pêche professionnelle en eau douce (C.N.A.P.P.E.D.).
} 
nouveaux marchés sur l'Asie du Sud-Est ${ }^{4}$. Une majeure partie de cette production est donc destinée à l'exportation. Ainsi, d'après les données disponibles, le commerce de l'anguille et de la civelle représente au total, en valeur, sur la moyenne des années 1995 à 1997, plus de 260 MF de chiffre d'affaires annuel à l'exportation (pour une production exportée constante de l'ordre de 1000 tonnes) ${ }^{5}$. Cette espèce se trouve ainsi placée au tout premier plan des espèces commerciales artisanales françaises au même titre que la sole, la baudroie ou la langoustine contribuant à hauteur d'environ $5 \%$ à $6 \%$ à la réduction du déficit structurel de la balance commerciale française des produits de la mer $^{6}$.

Bien qu'à l'analyse, ces chiffres doivent être maniés avec prudence en raison de la forte variabilité de l'abondance de la ressource d'une année sur l'autre et des difficultés inhérentes à la conjoncture économique dans les pays asiatiques qui laisse planer un doute sur la persistance d'une demande extérieure élevée et donc de prix soutenus, il demeure aujourd'hui que la pêche de l'anguille-civelle dans les estuaires en France pèse d'un poids crucial sur l'ensemble du secteur ainsi qu'au sein de la filière des pêches maritimes françaises où cette activité soutient en grande partie l'économie de la petite pêche côtière et conchylicole de nombreuses régions littorales.

En ce sens, cette espèce revêt donc un haut intérêt patrimonial et constitue un maillon essentiel de l'aménagement du territoire ainsi que pour la préservation des equilibres socio-économiques du littoral.

\section{INTRODUCTION}

Les marins-pêcheurs professionnels de la pêche dans les estuaires sont depuis longtemps sensibilisés aux exigences d'une protection renforcée et d'une gestion responsable des ressources amphihalines.

En effet, la pêche dans les estuaires se pratique dans des milieux fragiles et remarquables dont il paraît logique de réserver l'accès aux seuls petits métiers traditionnels ; elle se pratique aussi sur des espèces migratrices, qui, comme l'anguillecivelle, sont particulièrement sensibles à l'évolution de leur environnement et qu'il importe bien entendu de protéger de façon spécifique.

A la fin des années 1970, avec l'apparition des premiers signes d'une dégradation apparente de l'abondance des stocks d'anguilles à l'échelle européenne, -phénomène corroboré, dès cette époque, par un déclin marqué des apports totaux de captures, ${ }^{7}-$ les marins-pêcheurs des estuaires ont souhaité mettre en place, sous l'égide de l'ancien Comité interprofessionnel des poissons migrateurs et des estuaires (le C.I.P.E.), et ceci en liaison étroite avec le Ministère en charge de la pêche maritime, un système spécifique d'encadrement national de leurs activités de pêche sous la forme d'un régime contraignant de licences ${ }^{8}$.

\footnotetext{
"Principalement la R.P. de Chine, Hong-Kong, Taiwan pour un prix moyen de l'ordre de 1 ooo Frs/kg pour la civelle en 1997 ; le prix moyen était de lordre de $700 \mathrm{Frs} / \mathrm{kg}$ en $1998 / 1999$.

'Diverses sources: Douanes, Centre Français du Commerce Extérieur, Office nationai interprofessionnel des produits de la mer et de l'aquaculture (OFIMER).

"Cf, rapports annuels du commerce extérieur des produits de la mer du Fonds d'Intervention et d'Organisation des Marchés (F.I.O.M.)

"La production estimée passe de 1455 tonnes en 1976 (1 800 marins-pêcheurs) à 591 tonnes en 1983 (1 000 marins-pêcheurs) ; cf CASTELNAUD et al. (1994), p. 266, Tableau 1 : estimations de la production et du nombre de pêcheurs entre 1970 et 1986.

'Décision n' $1 / 79$ du Comité interprofessionnel des poissons migrateurs des estuaires (CIPE) du 8 février 1979 ; Commis 1978, sous l'égide du Comité Central des Pêches Maritimes (C.C.P.M.), le CIPE a été remplacé par une Commission spécialisee pour la pêche des poissons migrateurs et des estuaires (la C.I.P.E.) (cf, note supra).
} 
A travers le choix du système de la licence comme instrument central de gestion de l'effort de pêche dans les estuaires, la profession et les pouvoirs publics entendaient ainsi dès cette époque, en agissant tout à la fois sur la taille, la puissance, puis le nombre des navires admis à pêcher, privilégier la méthode de contrôle direct et préalable des moyens de production, de préférence à des mesures de limitation des captures autorisées au moyen de quotas jugées difficilement contrôlables dans un contexte de raréfaction de la ressource et en l'absence de toute infrastructure susceptible d'intervenir pour réguler l'évolution des marchés ${ }^{9}$.

D'abord limité à la seule pêche de la civelle, alevin de l'anguille, le système des " licences spéciales de pêche " (dit système des licences " CIPE ") s'est ensuite étenduà toutes les espèces de poissons amphihalins (anguilles, aloses, lamproies, truites de mer, saumons etc...) capturés par les marins-pêcheurs dans les estuaires et les zones maritimes côtières de la façade Atlantique et de la Manche-mer du Nord. Progressivement. il a été avantageusement complété par tout un arsenal de mesures de conservation additionnelles, adaptables selon les bassins d'exploitation, touchant aux dates d'ouveriure et de fermeture de la pêche ainsi qu'aux caraciéristiques des engins de capture ${ }^{10}$.

Aujourd'hui, le dispositif des licences «CIPE » tel qu'il est en vigueur dans le cadre de l'interprofession des pêches maritimes, se trouve au coeur de l'encadrement de l'efforl de pêche dans les estuaires : il permet tout à la fois de réserver l'accès aux ressources amphihalines au profit des seules petites unités de pêche du littoral ne dépassant pas certaines catégories de taille (12 mètres) et de puissance $(150 \mathrm{cv}$ ), de limiter très strictement le nombre de navires admis à pêcher tant au plan national (1 137 licences), qu'à l'échelle des principaux bassins hydrographiques (timbres « bassins »), et d'assurer un meilleur contrôle des activités de pêche dans les estuaires.

\section{MATÉRIEL ET MÉTHODES : VOIES ET MOYENS D'UNE GESTION RESPONSABLE DES POISSONS AMPHIHALINS AU MOYEN DE LICENCES}

En France, l'organisation des conditions d'exercice de la pêche maritime des poissons migrateurs et de la pêche dans les estuaires est fondée sur le principe de la limitation de l'accès à la ressource amphihaline et aux écosystèmes estuariens au moyen de licences délivrées par la profession sous le contrôle de l'administration, en l'occurrence le Ministère de l'Agriculture et de la Pêche au plan national et les services déconcentrés des Affaires maritimes aux niveaux régionaux et locaux.

La licence de pêche, en tant qu'outil de gestion de la ressource constitue une forme d'autorisation administrative préalable de pêche qui permet de réserver l'accès à certaines eaux ou le droit de pêcher certaines espèces de poissons, à une catégorie particulière de bénéficiaires.

La mise en place de ce régime de licences par les professionnels du secteur résulte ici d'une double habilitation législative et réglementaire tirée :

- d'une part, de la loi du 22 mai 1985 " relative à la pêche maritime " ${ }^{11}$ et dún décret du 25 janvier 1990 pris pour son application « fixant les conditions d'exercice dela

\footnotetext{
9 II n'existe pas d'organisation de producteurs pouvant faire jouer les mécanismes de l'organisation communted marchés (prix de retrait, normes de commercialisation) dans le secteur des poissons amphihalins.

${ }^{10}$ Ces mesures relèvent aujourd'hui le plus souvent de la compétence des Préfets de région (décret $n^{\circ}$ g0-94 $d x$ 25 janvier 1994 "fixant les conditions d'exercice de la pêche dans les eaux soumises à la réglementatith communautaire ", J.O.R.F. du 27 janvier 1990, p. 1152, et décret $n^{\circ}$ 94-158 du 16 février 1994 " relatif à la pécte des poissons vivant alternativement dans les eaux douces et les eaux salées ", J.O.R.F. du 23 février 1994. ${ }^{11}$ Loi $n^{\circ} 85-542$ du 22 mai 1985 * modifiant le décret du 9 janvier 1852 sur l'exercice de la pêche maritime J.O.R.F. du 24 mai 1985, p. 5815.
} 
pêche dans les eaux soumises à la réglementation communautaire ${ }^{12}$; ce texte prévoit formellement la possibilité pour le Ministre chargé de la mer de « soumettre, par arrêté, l'exercice de la pêche à un régime d'autorisation qui prend le nom de licences " (art. 10);

- d'autre part, de la loi du 2 mai 1991 et d'un décret du 30 mars $1992^{13}$ relatifs à lorganisation interprofessionnelle des pêches maritimes autorisant le Comité national ainsi que les Comités régionaux des pêches maritimes à prendre des délibérations dans le but de "la mise en adéquation (....) de l'outil de pêche avec la ressource disponible par linstitution et le contingentement de licences de pêche » (art. 4).

\section{RÉSULTATS : LES PRINCIPES ET MODALITÉS DE LA GESTION DES LICENCES PAR LA PROFESSION}

Les principes et les modalités de la gestion du dispositif des licences a pour la pêche des poissons migrateurs et pour la pêche dans les estuaires " aujourd'hui en vigueur en France sont définis par un arrêté ministériel du 15 septembre $1993^{14}$, complété par quatre délibérations adoptées chaque année par le Comité national des pêches maritimes et des élevages marins, sur proposition de sa Commission spécialisée pour la pêche dans les estuaires (C.I.P.E.) ${ }^{15}$.

Ces délibérations sont soumises à l'approbation du Ministre chargé des pêches maritimes qui les rend obligatoires. Elles revêtent alors un caractère de décision administrative exécutoire.

Profitant de l'opportunité de la réforme de l'organisation interprofessionnelle ayant conduit à la disparition du C.I.P.E. en $1992^{16}$, ces textes ont permis de reconduire, en le modernisant, le système des licences professionnelles introduit pour la première fois en 1979 à la suite de la création du C.I.P.E. A l'initiative de la profession, un certain nombre de changements importants sont alors intervenus, de manière à introduire, tant dans la gestion des droits de pêche que dans les procédures d'attribution, une dimension régionale à un dispositif d'encadrement jusqu'alors fortement centralisé.

\section{Une gestion des licences par bassins hydrographiques}

La licence « CIPE », ouvre, pour ses titulaires, le droit à l'exercice de la pêche des poissons migrateurs et de la pêche dans la zone maritime des estuaires ; toutefois, elle ne confère pas un droit général de pêche des poissons migrateurs à l'ensemble des estuaires français ; depuis la réforme mise en oeuvre en 1992, cette licence n'est plus valable, en principe, que pour un seul bassin hydrographique ou un seul groupe de rivières déterminé.

Désormais, le marin-pêcheur qui désire pêcher sur plusieurs bassins différents doit effectuer autant de demandes de licences que de bassins souhaités, la licence étant assortie de différents timbres " bassins » indiquant la zone de pêche pour laquelle elle est accordée.

La circonscription géographique de ces différents bassins et groupes de rivières est fixée par une délibération du C.N.P.M.E.M. (Figure 1).

\footnotetext{
"Décret $n^{\circ} 90-94$ du 25 janvier 1990 (op-cit).

${ }^{13}$ Décret $n^{\circ}$ 92-335 du 30 mars 1992 « fixant les règles d'organisation et de fonctionnement du Comité national des pêches maritimes et des élevages marins ainsi que des Comités régionaux et locaux des pêches maritimes ta des élevages marins ", J.O.R.F. du $1^{\text {or }}$ avril 1992, p. 4620.

"Arrêté du 15 septembre 1993 " instituant un régime commun de licences pour la pêche dans les estuaires et la peche des poissons migrateurs ", J.O.R.F. du 3 octobre 1993, p. 13770.

${ }^{15}$ Pour l'année 1999, voir les délibérations $n^{\circ} 13$ à 16/98 du Conseil du C.N.P.M.E.M. du 16 septembre 1998.

Loi $n^{\circ} 91-411$ du 2 mai 1991 (op-cit).
} 


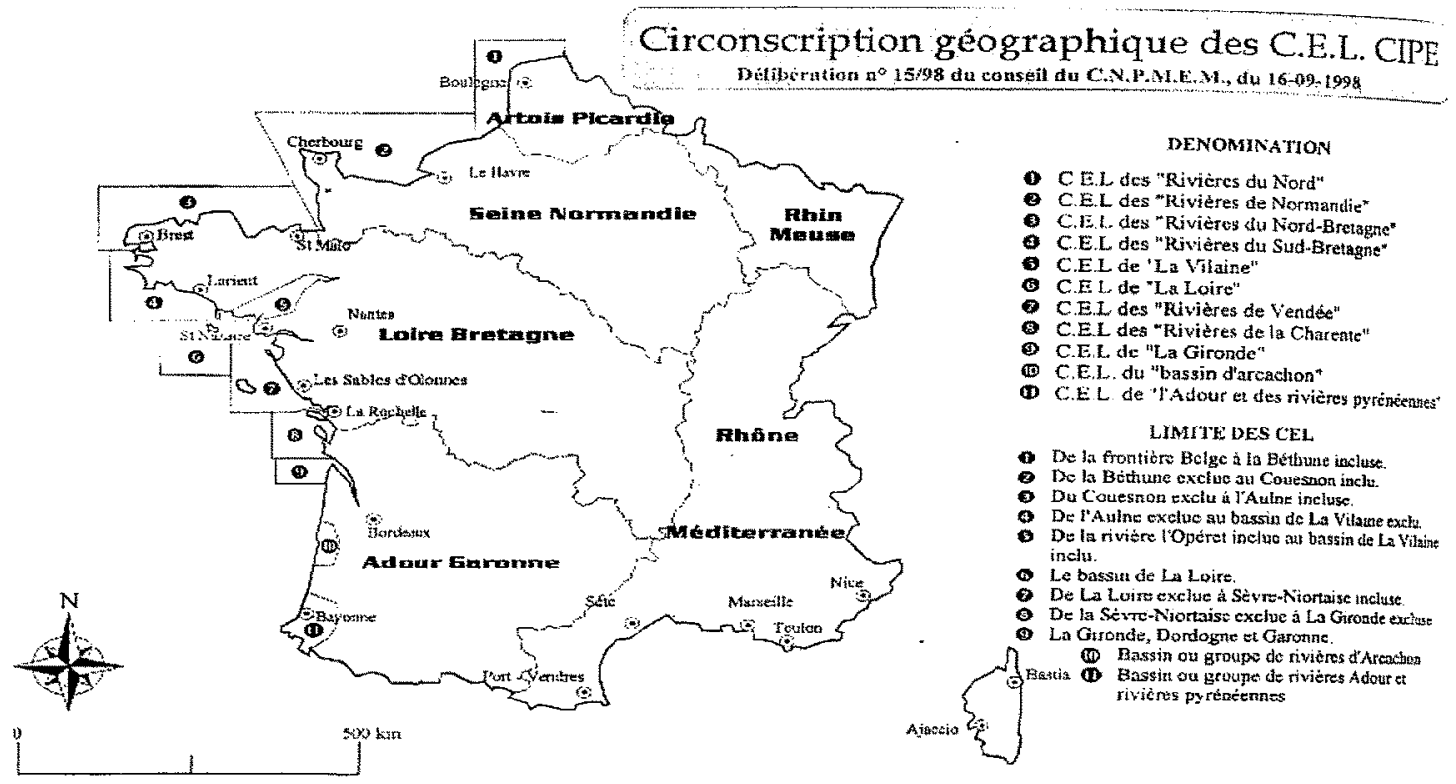

\section{Figure 1 \\ Circonscription des Commissions estuariennes de litiges.}

\section{Figure 1}

Geographical disputes areas of the estuary fishing commissions.

Ces « bassins " ou " groupes de rivières » constituent les unités de base pourla gestion du ou des droits de pêche conférés par la détention de la licence "CIPE".

Au nombre total de onze, répartis entre la frontière belge et la frontière espagnole, ces bassins ou groupes de rivières ont été regroupés de telle sorte à ne pas entrer en conflit avec la circonscription géographique des bassins hydrographiques français servant de cadre pour l'action des COmités de GEstion des POissons Mlgrateurs (CO.GE.PO.MI) ${ }^{17}$.

A l'échelle de chaque bassin, une Commission Estuarienne de Litiges (C.E.L). réélue chaque année est chargée de rendre les arbitrages nécessaires en cas de difficultés dans l'application de la réglementation professionnelle.

Présidées par un professionnel, ces Commissions de litiges délibèrent en présence d'un représentant de l'Administration des Affaires maritimes.

\section{Procédures de délivrance des licences}

Les demandes de licences sont instruites par les Comités locaux des pêches maritimes en étroite concertation avec les Chefs de quartiers des Affaires maritimes, chargés de vérifier la régularité des pièces figurant aux dossiers.

Après un examen éventuel des dossiers par la Commission estuarienne de litiges compétente, les licences sont ensuite délivrées par les Comités régionaux des pêches maritimes et des élevages marins.

\footnotetext{
17 Organismes de gestion des poissons migrateurs placés sous l'autorité des Préfets de région, coordinateurs de bassins; $c f$ décret $n^{\circ}$ 94-157 du 16 février 1994 "relatif à la pêche des poissons appartenant aux espèces vivar alternativement dans les eaux douces et dans les eaux salées », J.O.R.F. du 23 février 1994 , p. 3047.
} 
La licence est accordée uniquement pour un an ; elle est renouvelable, mais ne peut être ni cédée ni vendue de manière à éviter qu'elle n'entre dans le patrimoine privé de son détenteur créant ainsi une valeur économique à partir d'un droit à produire tiré d'une ressource rare et susceptible de susciter des transactions occultes incontrôlables ${ }^{18}$.

\section{Le contingentement des licences}

Le système des licences de pêche "CIPE " présente l'avantage de permettre un contrôle préalable de l'effort de pêche par une maîtrise de l'évolution numérique des flotilles autorisées à pêcher les poissons amphihalins.

Il appartient au C.N.P.M.E.M., sur proposition de la C.I.P.E., de fixer chaque année, par une délibération annuelle, les contingents de licences susceptibles d'être attribués par les Comités régionaux ainsi que la répartition de ces contingents entre les différents Comités locaux d'une même région (Tableau I).

Tableau I

Contingent des licences « CIPE 》 disponibles pour l'année 1999 (délibération

C.N.P.M.E.M. n¹4/98 du 16 septembre 1998). Table I

Restrictive licensing «CIPE» available for 1999.

\begin{tabular}{|c|c|c|}
\hline C.R.P.M.E.M. & C.L.P.M.E.M. & Contingent 1999 \\
\hline $\begin{array}{l}\text { NORD-PAS DE CALAIS } \\
\text { PICARDIE }\end{array}$ & BOULOGNE & 20 \\
\hline BASSE-NORMANDIE & $\begin{array}{c}\text { HONFLEUR } \\
\text { PORT-EN-BESSIN } \\
\text { GRANDCAMP / ISIGNY } \\
\text { EST-COTENTIN } \\
\text { OUEST-COTENTIN }\end{array}$ & $\begin{array}{l}19 \\
2 \\
6 \\
2 \\
3\end{array}$ \\
\hline BRETAGNE & $\begin{array}{c}\text { ST.MALO } \\
\text { ST.BRIEUC } \\
\text { PAIMPOL } \\
\text { NORD-FINISTERE } \\
\text { GUILVINEC } \\
\text { CONCARNEAU } \\
\text { LORIENT-ETEL } \\
\text { AURAY / VANNES }\end{array}$ & $\begin{array}{c}2 \\
11 \\
10 \\
21 \\
15 \\
7 \\
11 \\
127\end{array}$ \\
\hline PAYS-DE-LA-LOIRE & $\begin{array}{c}\text { LA TURBALLE } \\
\text { LOIRE ATLANT.SUD } \\
\text { NOIRMOUTIER } \\
\text { ST.GILLES-CROIX-DE-VIE } \\
\text { LES SABLES D'OLONNE }\end{array}$ & $\begin{array}{c}30 \\
195 \\
80 \\
38 \\
71 \\
\end{array}$ \\
\hline POITOU-CHARENTES & $\begin{array}{l}\text { LA ROCHELLE } \\
\text { MARENNES-OLERON }\end{array}$ & $\begin{array}{l}105 \\
155\end{array}$ \\
\hline AQUITAINE & $\begin{array}{l}\text { BORDEAUX } \\
\text { ARCACHON } \\
\text { BAYONNE }\end{array}$ & $\begin{array}{l}72 \\
65 \\
70 \\
\end{array}$ \\
\hline & TOTAL & 1137 \\
\hline
\end{tabular}

\footnotetext{
Le principe de la non-patrimonialisation des droits de pêche en mer est traditionnel aux pêches maritimes ; il est a carale par la loi d'orientation "sur la pêche maritime et les cultures marines " du 18 novembre 1997 qui énonce e caractère de bien collectif des ressources halieutiques (art. 4), J.O.R.F. du 19 novembre 1997, p. 16723. Voir aussi, débats à l'Assemblée nationale du 4 mars 1997, J.O.R.F. du 5 mars 1997, p.1590.
} 
Pour l'année 1999, le contingent national de licences «CIPE " a été fixé à 1137 unités ; il est très important de souligner ici que ce chiffre demeure inchangé depuis 1995 : il correspond donc à un «gel » du nombre de licences décidé par la profession al plan national en tenant compte de 3 critères distincts, conformément aux exigences de l'arrêté ministériel du 15 septembre 1993 :

- les capacités biologiques dans les eaux concernées ;

- les caractéristiques des navires;

- les antériorités de pêche.

En fait, si la situation inquiétante de certains stocks de poissons amphihalins (en particulier de l'anguille) a permis de justifier -voici déjà 5 ans- le principe de la limitationdu nombre de licences aux yeux de la profession, seuls les deux derniers critères lles caractéristiques des navires et les antériorités de pêche) ont véritablement joué un rôle prépondérant pour la fixation des niveaux de contingents de licences actuellement disponibles.

Cette décision en faveur du gel de la flottille relève d'une démarche de précaution dans une optique de pêche responsable et constitue un signe bien tangible de la volonlé des professionnels du secteur à ne pas permettre une augmentation de l'effort de pêche dans les estuaires au-delà des pratiques de pêche existantes.

\section{Le champ d'application de la licence CIPE}

* Au plan matériel : la licence « CIPE » est attribuée conjointement au pairon propriétaire embarqué (et à son navire), titulaire d'un rôle d'équipage à la pêche et sous réserve du respect de certaines conditions très strictes en termes de professionnalisme du demandeur et de limitation de la taille et de la puissance du navire.

C'est ainsi que pour éviter les comportements opportunistes liés à la saisonnalitéde certaines pêcheries, le demandeur de la licence doit pouvoir justifier devant l'administration, d'un temps de navigation de 36 mois à la pêche ( 24 mois pour les capacitaires) et avoir pratiqué la pêche professionnelle 9 mois dans l'année ; quant aux conditions tenant aux caractéristiques des navires, elles permettent d'éviter l'entrée de grosses unités en provenance de la mer ouverte dans les estuaires: seuls sont admis les navires d'un tonnage et d'une longueur respectivement inférieurs à 10 tonneaux de jaug: brute et à 12 mètres (10 mètres dans le bassin de la Vilaine) et dont la puissance des moteurs ne peut excéder $150 \mathrm{cv}^{19}$.

* Au plan géographique : la licence “ CIPE » s'applique sur l'ensemble du littorl français, entre la frontière belge et la frontière espagnole, à l'exclusion de la mer Méditerranée, à toutes les espèces présentes dans les estuaires entre la linite transversale de la mer et la limite de salure de l'eau 20 et aux seules espéces amphihalines, en mer ouverte, jusqu'à la limite extérieure des eaux territoriales ${ }^{*}$ (Figure 2).

\footnotetext{
19 Cette puissance est réduite à $100 \mathrm{cV}$ pour la pêche des aloses, anguilles, lamproies, saumons et truites dent 20 La limite de salure de l'eau marque la séparation à l'intérieur des fleuves et rivières entre les eaux sounises la réglementation maritime et les eaux soumises à la réglementation fluviale ; la limite transversaie de la ms marque le long des côtes la ligne de partage entre le domaine public maritime et le domaine public flimat (DUFAU, 1977).

${ }^{21}$ D'une largeur pouvant aller jusqu'à 12 milles marins.
} 

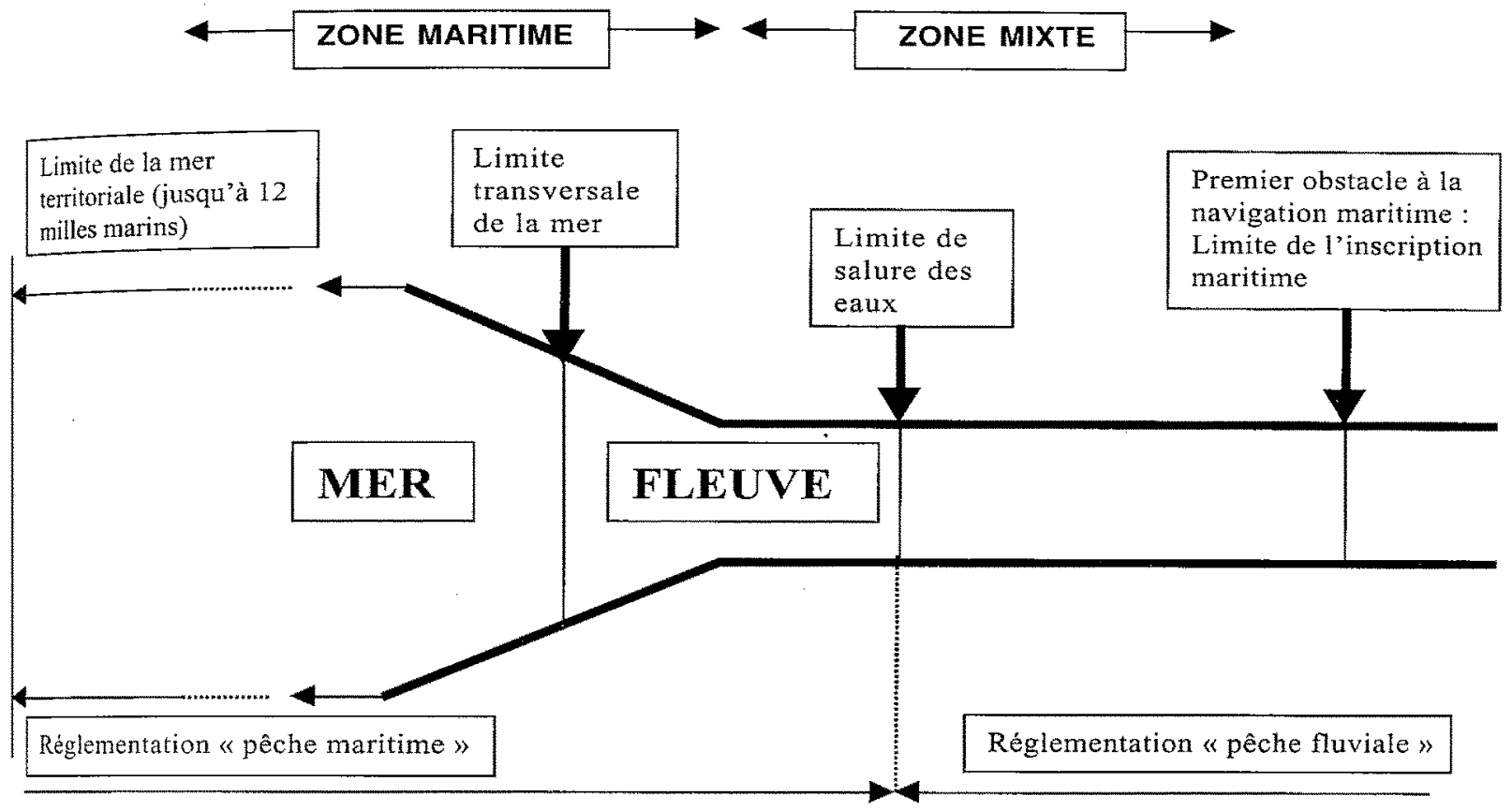

Figure 2

Limites administratives de l'espace géographique de la pêche en estuaire.

Figure 2

Administrative borderlines of the geographic area of estuary fishing.

\section{DISCUSSION : INTÉRÊT D'UN ENCADREMENT STRICT DES PÊCHERIES DE POISSONS MIGRATEURS AU MOYEN DE LICENCES}

\section{Une meilleure protection des écosystèmes estuariens}

Le système des licences « CIPE " correspond à un encadrement très strict de l'effort de pêche des poissons migrateurs et de pêche dans la zone maritime des estuaires en France, tant au plan national, qu'au niveau de chaque bassin hydrographique ; désormais, seul un nombre limité -et contingenté- de marins-pêcheurs professionnels spécialisés et de navires de petite dimension extrêmement dépendants de l'activité de pêche peuvent avoir accès aux zones et aux espèces sensibles des estuaires.

De ce point de vue, son intérêt est évident au regard des impératifs d'une exploitation rationnelle et durable des ressources amphihalines, qui soit à la fois respectueuse des écosystèmes estuariens et garante de la rentabilité économique de ces petites entreprises de pêche dont le maintien dans l'activité est absolument vital pour la préservation des équilibres socio-économiques du littoral.

Plus largement, le dispositif a aussi fait la preuve de son efficacité pour éviter lintrusion de gros navires dans les zones côtières des estuaires qui constituent autant de leux privilégiés pour le développement de frayères et de nurseries où se reproduisent et grandissent de nombreuses espèces de poissons et crustacés avant de rejoindre la mer 


\section{Un instrument au service de la lutte anti-braconnage}

Dans les années récentes, le système des licences «CIPE " a aussi favorisé la mise en évidence de l'existence d'un braconnage important de la civelle dans les estuaires par des pêcheurs à pied dépourvus de toute autorisation pour pratiquer cette activité à titre professionnel. Endémique voici 4 ou 5 ans, ce phénomène, qui est une source de concurrence déloyale pour les marins-pêcheurs et de pression de pêche accrue sur la ressource, semble devoir se résorber peu à peu même s'il subsiste encore, faute de moyens de contrôle matériels et humains en nombre suffisant, quelques poches de résistance tenaces en certains points du littoral.

Sous l'action de la profession, qui ne cesse de tirer régulièrement le signal d'alarme, les pouvoirs publics, et plus récemment les tribunaux, ont en effet pris conscience de l'importance de certains réseaux de commercialisation occulte de la civelle, qui tirent profit d'une ressource naturelle, sans la moindre contrepartie pour la collectivité tout en déstabilisant les vrais pêcheurs professionnels organisés, respectueux de leurs obligations fiscales et sociales et soucieux de préserver le patrimoine halieutique et les milieux naturels.

\section{Une reconnaissance de la spécificité des pêcheries estuariennes}

La mise en place d'un encadrement particulier pour la pêche dans les estuaires el pour la pêche des poissons migrateurs a aussi permis de sauvegarder le caractère propre de ces pêcheries au sein d'un environnement économique et institutionnel extrêmement sensible aux conflits d'intérêts très souvent contradictoires entre les aménageurs d'espace et les protecteurs de la nature dans ces zones de passages obligés et d'interfaces fragiles que sont les estuaires. Acteurs reconnus de l'exploitation des ressources de poissons migrateurs, les marins-pêcheurs des estuaires, clairement identifiés en qualité de titulaires de la licence «CIPE » disposant de l'exclusivité du droit de pêche, sont aussi des témoins privilégiés de l'évolution des milieux estuariens et de la qualité des eaux à l'embouchure des fleuves et des cours d'eau.

Car, aujourd'hui, le principal enjeu de l'avenir des pêcheries d'estuaires n'est pas seulement dans le développement d'une pratique de pêche responsable, ni même dans la poursuite d'une lutte efficace contre le braconnage et les pratiques de commercialisation illicite de la civelle, il est avant tout d'ordre écologique et dépasse les frontières d'un seul pays.

En effet, selon le dernier rapport du Conseil International pour l'Exploration de la Mer (C.I.E.M.) ${ }^{22}$, les populations d'anguilles européennes continuent à présenter, selonles dernières évaluations scientifiques en matière de recrutement, des signes très sérieux d'une dégradation apparente.

Les marins-pêcheurs professionnels sont parfaitement conscients des effets négatifs d'un tel phénomène qui, à plus ou moins long terme, peut gravement mettre en péril la durabilité de leurs propres activités économiques.

Toutefois, il ne saurait être question, à leurs yeux, de considérer que l'exploitation halieutique et la mortalité due à la pêche, sont les seuls facteurs responsables d'une telle situation.

${ }_{22}$ Cf rapport du C.I.E.M. à la Commission européenne (extrait rapport de l'A.C.F.M.-C.I.E.M.- mai 1998, 14 p). 
Comme le montrent de nombreuses études et travaux techniques ou scientifiques disponibles (voir notamment VIGNEUX, 1994), bien d'autres causes, exogènes à l'activité de pêche stricto-sensu, doivent être recherchées, à commencer par l'impact des activités humaines en général sur la dégradation des milieux aquatiques et des habitats naturels (effets des pollutions, dragages, pompages, constructions de digues, de chenaux, de barrages etc...), ou encore l'impact généré par certains phénomènes hydro-climatiques (niveaux des précipitations, des températures, variation des courants marins etc...).

Nul ne nie que tous ces éléments, auxquels il faudrait ajouter l'influence de certaines pathologies propres à l'espèce ${ }^{23}$, peuvent affecter, de façon considérable, la dynamique des stocks et l'abondance de l'anguille-çivelle d'une année sur l'autre.

Dans ces conditions, seul une action de grande envergure au plan international, dépassant la sphère d'intervention d'un seul Etat et agissant sur l'ensemble des acteurs et des causes de la dégradation des stocks seraient susceptibles de produire des résultats.

L'enjeu est de taille pour la profession : il en va de la sauvegarde du caractère propre des pêcheries spécifiques d'estuaires et de poissons migrateurs, toutes parfaitement dignes d'intérêt aux plans économique, social, écologique et culturel au même titre que les entreprises et les hommes qui en vivent.

\section{BIBLIOGRAPHIE}

BRUSLE J., 1994. L'anguille européenne : un poisson sensible aux stress environnementaux et vulnérable à diverses atteintes pathogènes. Bull. Fr. Pêche Piscic., 335, 237-260.

CASTELNAUD G., GUERAULT D., DESAUnAY Y., EliE P., 1994. Production et abondance de la civelle en France au début des années 1990. Bull. Fr. Pêche Piscic., 335, 263-287.

COLLECTIF, 1991. Actes des premières Assises nationales des pêcheurs professionnels. Bayonne, 17, 18 et 19 octobre 1991.226 p.

COLLECTIF, 1992. Actes des secondes Assises nationales de la pêche professionnelle. Nantes, 3 et 4 novembre 1992. 109 p.

COLLECTIF, 1993. Actes des troisièmes Assises nationales de la pêche professionnelle. Carsac, 22, 23 et 24 septembre 1993. 104 p.

COLLECTIF, 1996. Actes des quatrièmes Assises nationales de la pêche professionnelle continentale et estuarienne. Libourne, 16, 17 et 18 octobre $1996.80 \mathrm{p}$.

DUFAU J., 1977. Le domaine public. Edition du Moniteur.

MORIARTY C., 1996. The European eel fishery in 1993 and 1994. 1er rapport d'un groupe de travail financé par l'Union européenne (action concertée AIR, $n^{\circ}$ A94-1939). Fisheries bulletin $n^{\circ} 14$, Marine Institute DUBLIN, $52 \mathrm{p}$.

MORIARTY C., DEKKER W., 1997. Management of the European eel. $2^{\text {eme }}$ rapport d'un groupe de travail financé par l'Union européenne (action concertée AIR, $n^{\circ}$ A941939). Fisheries bulletin no 15, Marine Institute DUBLIN, $110 \mathrm{p}$.

VIGNEUX E. (ed.), 1994. Spécial anguille (volume 1), bilan des travaux et expérimentations en cours. Bull. Fr. Pêche Piscic., 335, $328 \mathrm{p}$.

\footnotetext{
EX: parasite "Anguillicola " qui pourrait affecter la capacité des géniteurs à regagner leur lieu de ponte
BRUSLE, 1994).
} 